\title{
Publisher's Note: Enhanced Tunneling Electroresistance in Ferroelectric Tunnel Junctions due to the Reversible Metallization of the Barrier [Phys. Rev. Lett. 116, 197602 (2016)]
}

Xiaohui Liu, J. D. Burton, and Evgeny Y. Tsymbal

(Received 13 May 2016; published 20 May 2016)

DOI: 10.1103/PhysRevLett.116.209902

This Letter was published online on 11 May 2016 with typographical errors in the text on page 4. On page 4, left-hand column, the first sentence of the first paragraph should read as "Our calculations confirm that the tunnel junction indeed exhibits much lower tunneling resistance with polarization pointing into $n$ - $\mathrm{SrTiO}_{3}$." The sixteenth line of the same paragraph should read as "on resistance ratio is about $2.3 \times 10^{3}$." The Letter has been corrected as of 13 May 2016. The text is correct in the printed version of the journal. 